\title{
miR-101-3p sensitizes lung adenocarcinoma cells to irradiation via targeting BIRC5
}

\author{
XIN MENG ${ }^{1}$, YANFEI SUN ${ }^{2}$, SHIYING LIU ${ }^{3}$ and YANCHAO MU Y $^{4}$ \\ ${ }^{1}$ Department of Hyperbaric Oxygen and ${ }^{2}$ Trauma Center/Burn Ward, The First Affiliated Hospital of \\ China Medical University; ${ }^{3}$ School of Nursing, China Medical University, Liaoning, Shenyang 110001; \\ ${ }^{4}$ Department of Laboratory Medicine, Anyang Maternity and Child Healthcare Hospital, Anyang, Henan 455000, P.R. China
}

Received August 11, 2020; Accepted January 4, 2021

DOI: $10.3892 / \mathrm{ol} .2021 .12543$

\begin{abstract}
Lung adenocarcinoma (LUAD) has been considered as the most common cause of cancer-associated mortality. Radiotherapy resistance is one of the main reasons for LUAD treatment failure. The microRNA (miR)-101-3p has been previously reported to function as a tumor suppressor in several types of cancer, including LUAD. The present study aimed to explore the role and mechanism of miR-101-3p on radioresistance of lung adenocarcinoma cells through bioinformatics analysis and biological experiments. Based on the analysis of Gene Expression Omnibus (GEO) and The Cancer Genome Atlas (TCGA) data, it was demonstrated that the expression of miR-101-3p was low in LUAD tissues compared with normal lung tissues and was associated with poor prognosis of patients with LUAD. The results of the CCK-8 assay, colony formation assay, immunofluorescence staining, caspase- 3 activity assay and western blotting demonstrated that miR-101-3p overexpression sensitized LUAD cells to ionizing radiation by decreasing the abilities of LUAD cell proliferation, colony formation, DNA damage repair and increasing caspase-3 activity and apoptosis of LUAD cells following ionizing radiation. Furthermore, according to bioinformatics analysis and luciferase assay, baculoviral IAP repeat containing 5 (BIRC5) was identified as a direct target of miR-101-3p. Increased BIRC5 expression reversed the miR-101-3p-mediated increase in LUAD cell radiotherapy sensitivity. Taken together, the results of the present study demonstrated that miR-101-3p may be considered as a potential target that can enhance LUAD cell sensitivity to radiotherapy, which may provide a new strategy to improve therapy in patients with LUAD.
\end{abstract}

Correspondence to: Dr Yanfei Sun, Trauma Center/Burn Ward, The First Affiliated Hospital of China Medical University, 155 Nanjing Street, Liaoning, Shenyang 110001, P.R. China

E-mail: cmuyanfeisun@163.com

Key words: miR-101-3p, lung adenocarcinoma, prognosis, radiosensitivity, baculoviral IAP repeat containing 5

\section{Introduction}

Lung cancer is still the leading cause of cancer-related deaths due to the high diagnosis rate and mortality rate (1). In the United States, it is estimated that 225,000 people are diagnosed with lung cancer and 160,000 people die of lung cancer each year (1). Histologically, non-small cell lung cancer (NSCLC) is the main subtype of lung cancer, and lung adenocarcinoma (LUAD) is the most diagnosed type of NSCLC (1). Although the molecular mechanisms of LUAD have been elucidated and treatments for LUAD have improved, the overall survival of patients with LUAD remains poor (2). For these patients, radiotherapy is considered as a promising treatment strategy that can prolong patient survival and improve the quality of life. However, radiotherapy resistance is a critical issue limiting the efficacy of radiation therapy (3). It is therefore urgent to clarify the underlying mechanisms of tumor cell radioresistance and increase the tumor sensitivity to radiotherapy.

MicroRNAs (miRNA) represent a group of short non-coding RNAs that regulate gene expression by complementary base pairing with the 3'-UTR of mRNA and trigger translation repression or RNA degradation (4). Over the past decades, miRNAs have been demonstrated to serve critical roles in numerous types of cancer, and their dysregulation is closely associated with certain tumorigenic processes, such as proliferation, apoptosis, cell cycle regulation and stress response $(5,6)$. In addition, evidence has shown that miRNAs can modulate cancer cell radiosensitivity $(7,8)$, which indicates their potential for improving the efficacy of radiotherapy. For example, miR-101-3p expression has been demonstrated to be decreased in tumor tissues or cells and involved in the regulation of various cancer activities (9-11). Wu et al (9) reported that $\mathrm{miR}-101-3 \mathrm{p}$ could target the serum response factor and inhibit HOX transcript antisense RNA-mediated proliferation and invasion of gastric carcinoma cells. In addition, Li et al (11) reported that miR-101-3p can enhance the sensitivity of bladder urothelial carcinoma to cisplatin by silencing the expression of Enhancer of zeste homolog 2. However, the biological function and underlying mechanisms of miR-101-3p in LUAD remain unclear.

Baculoviral IAP repeat containing 5 (BIRC5), also known as survivin, belongs to the inhibitor of apoptosis protein (IAP) family, and blocks apoptosis induced by various stimuli, 
such as radiation and chemical drugs (12). The abnormal amplification of BIRC5 protein has been found in numerous malignancies, including breast cancer, colon carcinomas and melanoma $(13,14)$. Furthermore, BIRC5 overexpression has been demonstrated to be closely associated with tumor initiation and development, radiotherapy and chemotherapy resistance and poor prognosis in patients with cancer, such as glioblastoma and head and neck squamous cell cancer $(15,16)$. In the last decades, BIRC5 has attracted considerable attention as a therapeutic target for anticancer strategies due to its role in regulating the sensitivity of cancer cells to radiotherapy and chemotherapy $(17,18)$. Therefore, it is crucial to elucidate the mechanism by which BIRC5 regulates human cancer sensitivity to radiotherapy and chemotherapy.

The present study aimed to elucidate the effect of miR-101-3p on the radiosensitivity of LUAD cells and to explore whether miR-101-3p could affect this radiosensitivity by directly regulating BIRC5. The findings from this study may provide some future perspectives for the radio-sensitization of LUAD.

\section{Materials and methods}

Bioinformatics analysis. The RNA array datasets [GSE48414 (19) and GSE74190 (20)] were downloaded from the Genome Expression Omnibus database (www.ncbi.nlm. nih.gov/gds). Furthermore, RNA seq data and clinical survival data of LUAD patients extracted from The Cancer Genome Atlas (TCGA) database (https://portal.gdc.cancer.gov/) were used to investigate the expression level of miR-101-3p and BIRC5 in LUAD tissues and normal adjacent tissues and survival of LUAD patients. The present study, used Starbase (http://starbase.sysu.edu.cn/) (21) to predict the target gene of miR-101-3p. The online Kaplan-Meier analysis of the survival of all patients with lung cancer which accepted radiotherapy with different BIRC5 expression levels was assessed using the Kaplan-Meier Plotter (http://kmplot.com/analysis/index.php? $\mathrm{p}=$ service \&cancer=lung) (22).

Cell culture. The LUAD cell lines Calu3, H1299 and H292 and the normal lung epithelial cell line BEAS-2B were purchased from the American Type Culture Collection. BEAS-2B cell line was cultured in BEBM medium (Lonza Group, Ltd.) whereas Calu3, H1299 and H292 cell lines were cultured in RPMI-1640 medium (Gibco; Thermo Fisher Scientific, Inc.) supplemented with 10\% FBS (HyClone; GE Healthcare Life Sciences), $100 \mu \mathrm{g} / \mathrm{ml}$ streptomycin and $100 \mathrm{U} / \mathrm{ml}$ penicillin (Gibco; Thermo Fisher Scientific, Inc.). All cell lines were placed at $37^{\circ} \mathrm{C}$ in a humidified incubator containing $5 \% \mathrm{CO}_{2}$.

Cell transfection. The plasmids pcDNA3.1-BIRC5 and pcDNA3.1-Vector and the miR-101-3p mimics and negative control (NC) mimics were purchased from Guangzhou RiboBio Co., Ltd. Calu3 and H292 cells were seeded in 6 -well plates $\left(8 \times 10^{5}\right.$ cells/well) and transfected with $1.6 \mu \mathrm{g}$ of pcDNA3.1-BIRC5/vector plasmids or $50 \mathrm{nM} \mathrm{mimics/NC}$ mimics using Lipofectamine ${ }^{\circledR} 2000$ (Invitrogen; Thermo Fisher Scientific, Inc.). The media in each well was then replaced with fresh medium $6 \mathrm{~h}$ following incubation with the transfection mixture at $37^{\circ} \mathrm{C}$. After $48 \mathrm{~h}$, the cells were subjected to subsequent analyses.

CCK-8 assay. Cells were seeded into 96-well plates at the density of $2 \times 10^{3}$ cells per well and placed at $37^{\circ} \mathrm{C}$ in a humidified incubator containing $5 \% \mathrm{CO}_{2}$ for $12 \mathrm{~h}$. Subsequently, cells were treated with various doses of ionizing radiation (IR; 0, 2, 4, 6 and $8 \mathrm{~Gy}$ ). After another $72 \mathrm{~h}$ of incubation post-irradiation, the cell survival rate was assessed using Cell Counting Kit- 8 (CCK-8; Signalway Antibody LLC) assay. Cells were incubated with $20 \mu \mathrm{lCCK}-8$ reagent at $37^{\circ} \mathrm{C}$ for $4 \mathrm{~h}$. Absorbance was detected at $450 \mathrm{~nm}$ using a microplate reader.

Colony formation assay. Colony formation assay was performed to evaluate cell sensitivity to radiation. Briefly, following transfection for $48 \mathrm{~h}$, cells were seeded in 6-well plates at an appropriate number of cells $(200,400,800,1,500$ and 3,000 cells/well) and cultured for $12 \mathrm{~h}$. Subsequently, cells were treated with various doses of IR $(0,2,4,6$ and $8 \mathrm{~Gy})$ and cultured for 12 days. Cells were then fixed with $100 \%$ methanol at room temperature for $10 \mathrm{~min}$ and stained with $0.5 \%$ crystal violet at room temperature for $15 \mathrm{~min}$. Colonies $>50$ cells were subsequently imaged using a light microscope and data were analyzed using SPSS (SPSS, Inc.; version 13.0).

Caspase-3 activity. Following transfection for $48 \mathrm{~h}$, cells were treated with a dose of 0 or 6 Gy IR. After $48 \mathrm{~h}$, the activity of caspase-3 was assessed in cells using the Caspase-3 Assay kit (Sigma-Aldrich; Merck $\mathrm{KGaA}$ ) according to the manufacturers' instructions.

$R N A$ extraction and reverse transcription-quantitative (RT-q) $P C R$. Total RNA was isolated from cultured cells using TRIzol $^{\circledR}$ (Invitrogen; Thermo Fisher Scientific, Inc.) according to the manufacturers' instructions. The cDNAs were obtained using a Reverse Transcription kit (Takara Biotechnology Co., Ltd.). The temperature protocol for the reverse transcription reaction consisted of cDNA synthesis at $37^{\circ} \mathrm{C}$ for $60 \mathrm{~min}$ and termination at $80^{\circ} \mathrm{C}$ for $2 \mathrm{~min}$. RT-qPCR reactions were performed on the ABI Prism 7900 system (Thermo Fisher Scientific, Inc.) using SYBR-Green PCR kit (Thermo Fisher Scientific, Inc.). The thermocycling conditions were as follows: Initial denaturation at $95^{\circ} \mathrm{C}$ for $2 \mathrm{~min}$, followed by 39 cycles at $94^{\circ} \mathrm{C}$ for $20 \mathrm{sec}$ and $60^{\circ} \mathrm{C}$ for $30 \mathrm{sec}$, and final extension at $72^{\circ} \mathrm{C}$ for $30 \mathrm{sec}$. U6 and GAPDH were used as the internal controls. The sequences of the primers were as follows: miR-101-3p, forward 5'-ACGGGCGAGCTACAG TACTGTG-3', reverse 5'-CCAGTGCAGGGTCCGAGGTA-3'; BIRC5, forward 5'-AGGACCACCGCATCTCTACAT-3', reverse 5'-AAGTCTGGCTCGTTCTCAGTG -3'; U6, forward 5'-TGCGTTCCCTTTGTCATCCT-3', reverse 5'-AACGCT TCACGAATTTGCGT-3'; and GAPDH, forward 5'-AATCCC ATCACCATCTTC-3' and 5'-AGGCTGTTGTCATACTTC-3'. The relative expression levels were normalized to endogenous controls and were expressed as $2^{-\Delta \Delta \mathrm{Cq}}(23)$.

Immunofluorescence staining. After Calu3 and H292 cells $\left(4 \times 10^{4}\right.$ cells) were incubated on 24 -well coverslips (Thermo Fisher Scientific, Inc.) overnight at $37^{\circ} \mathrm{C}$, cells were treated with 6 Gy IR. After 24 h, cells were fixed with 
$4 \%$ paraformaldehyde at room temperature for $1 \mathrm{~min}$ and with ice-cold methanol at room temperature for $10 \mathrm{~min}$. Permeabilization was performed using $0.2 \%$ Triton X-100 in PBS-T (0.05\% Tween-20 in PBS) for 10 min followed by blocking with $5 \%$ bovine serum albumin (Gibco; Thermo Fisher Scientific, Inc.) in PBS-T at room temperature for $1 \mathrm{~h}$. Cells were then incubated with the primary antibody against $\gamma-\mathrm{H} 2 \mathrm{~A}$ histone family member $\mathrm{X}[\gamma-\mathrm{H} 2 \mathrm{AX}$, a biomarker for DNA double-strand breaks (DSBs) (24); 1:500; cat. no. 9718; Cell Signaling Technology, Inc.] overnight at $4^{\circ} \mathrm{C}$, followed by the appropriate Alexa fluor ${ }^{\circledR}$-labeled secondary antibodies (1:400; cat. no. 4412; Cell Signaling Technology, Inc.) at room temperature for $1 \mathrm{~h}$. Coverslips were mounted with ProLong Diamond Anti-fade reagent and DAPI (Invitrogen; Thermo Fisher Scientific, Inc.). Cells were imaged using a laser scanning microscope (Axio Imager.Z2; Zeiss $\mathrm{GmbH}$ ).

Western blotting. The LUAD cell lines were lysed using RIPA lysis buffer (Thermo Fisher Scientific, Inc.) and protein concentration was determined with the BCA assay kit (Beyotime Institute of Biotechnology). Proteins ( $40 \mu \mathrm{g} /$ well) were separated by $12 \%$ SDS-PAGE and transferred onto PVDF membranes (EMD Millipore). Membranes were blocked with $5 \%$ skimmed milk at room temperature for $1 \mathrm{~h}$ and were incubated with primary antibodies against $\gamma$-H2AX (1:1,000; cat. no. 9718; Cell Signaling Technology, Inc.), $\operatorname{Bax}(1: 2,000$; cat. no. 60267-1-Ig; ProteinTech Group, Inc.), Bcl2 (1:1,000; cat. no. 15071; Cell Signaling Technology, Inc.), GAPDH (1:2,000; cat. no. 5174; Cell Signaling Technology, Inc.) and BIRC5 (1:2,000; cat. no. ab469; Abcam) overnight at $4^{\circ} \mathrm{C}$. Membranes were then incubated with a horseradish peroxidase-conjugated goat anti-rabbit secondary antibody (1:5,000; cat. no. ab6721; Abcam) at room temperature for $1 \mathrm{~h}$. Enhanced chemiluminescence (ECL, Abcam) reagent was used to detect the signal on the membrane. The data were analyzed via densitometry using ImageJ software version 1.41; (National Institutes of Health) and normalized to expression of the internal control GAPDH.

Luciferase reporter assay. A luciferase reporter assay was performed to verify whether BIRC5 was a direct target of miR-101-3p. Cells were co-transfected with luciferase reporter plasmid PGL3-WT-BIRC5/PGL3-MUT-BIRC5 (Shanghai GenePharma Co., Ltd.) and miR-101-3p mimic (5'-UACAGU ACUGUGAUAAUCGAA-3') and miR-NC (5'-CAGUAC UUUUGUGUAGUACAA-3') using Lipofectamine ${ }^{\circledR} 2000$ (Invitrogen; Thermo Fisher Scientific Inc.) at $37^{\circ} \mathrm{C}$ for $48 \mathrm{~h}$. The luciferase and Renilla signals were then measured using the Dual Luciferase Reporter Assay kit (Promega Corporation) according to the manufacturer's instructions. The luciferase activity was normalized using Renilla activity. The automatic microplate reader (Molecular Devices, LLC) was used in luciferase assays detection.

Statistical analysis. Statistical analyses were performed using SPSS (IBM Corp.; version 20.0). Data are presented as the mean \pm standard deviation of three independent experiments. Log-rank test was used to determine the statistical significance of Kaplan-Meier overall survival data of patients with LUAD. An unpaired t-test was used to compare data between two groups whereas one-way ANOVA followed by Tukey's or Bonferroni's post hoc test was used to compare data between three groups or more. The gene expression correlation between miR-101-3p and BIRC5 was assessed using the Pearson's correlation test. $\mathrm{P}<0.05$ was considered to indicate a statistically significant difference.

\section{Results}

miR-101-3p expression is lower in patients with LUAD and associated with poor prognosis. The expression of miR-101-3p was investigated in LUAD and adjacent tissues using NCBI/GEO data mining and TCGA database analysis. As presented in Fig. 1A-C, miR-101-3p expression was significantly lower in LUAD tissues compared with adjacent tissues. Furthermore, all TCGA/LUAD patients were separated into low and high expression groups based on median value of miR-101-3p (10468.29) expression, and it was revealed that patients with low expression of miR-101-3p in TCGA/LUAD had a poorer prognosis compared with patients with high miR-101-3p expression (Fig. 1D). In addition, miR-101-3p expression was determined by RT-qPCR in the normal lung epithelial cell line BEAS-2B and the three LUAD cell lines Calu3, H1299 and H292. As shown in Fig. 1E, the expression of miR-101-3p in the three LUAD cell lines was significantly lower compared with the BEAS-2B cell line. These results suggested that miR-101-3p expression was lower in LUAD tissues compared with normal lung tissues and was associated with poor prognosis of patients with LUAD.

miR-101-3p overexpression sensitizes LUAD cells to IR. The two LUAD cell lines Calu3 and H292 were chosen for subsequent experiments, as they exhibited the lowest expression of miR-101-3p, and were transfected with miR-101-3p mimic or miR-NC. As presented in Fig. 2A, the expression of miR-101-3p in both cell lines was significantly increased compared with the control group $48 \mathrm{~h}$ following transfection. Subsequently, the role of $\mathrm{miR}-101-3 \mathrm{p}$ on the radiosensitivity was examined. The results from CCK-8 assay demonstrated a decreased proliferation LUAD cells overexpressing miR-101-3p (Fig. 2B and C). In addition, the results from colony formation assay showed that miR-101-3p overexpression significantly enhanced the radiosensitivity of LUAD cells (Fig. 2D-F).

The effect of IR on cancer cell death mainly depends on DNA double-strand breaks (DSBs), and repair of this DNA damage determines the radiosensitivity of cancer cells (25). To investigate whether miR-101-3p could affect DNA damage repair ability, the expression of $\gamma-\mathrm{H} 2 \mathrm{AX}$, which is a biomarker for DSBs, was determined. The results from immunofluorescence staining demonstrated that more foci of $\gamma$-H2AX were observed in cells overexpressing miR-101-3p compared with the control group cells at $24 \mathrm{~h}$ following irradiation (Fig. 2G). Furthermore, the results from western blotting indicated that $\gamma$-H2AX expression in cells overexpressing miR-101-3p was significantly increased compared with the control group at $24 \mathrm{~h}$ following irradiation (Fig. 2J). In addition, to evaluate the effect of miR-101-3p on cell apoptosis, a caspase-3 assay was performed to detect changes in caspase-3 activity after irradiation treatment. As presented in Fig. $2 \mathrm{H}$ and $\mathrm{I}$, the activity of caspase- 3 in cells overexpressing miR-101-3p was significantly higher compared with the control group cells 

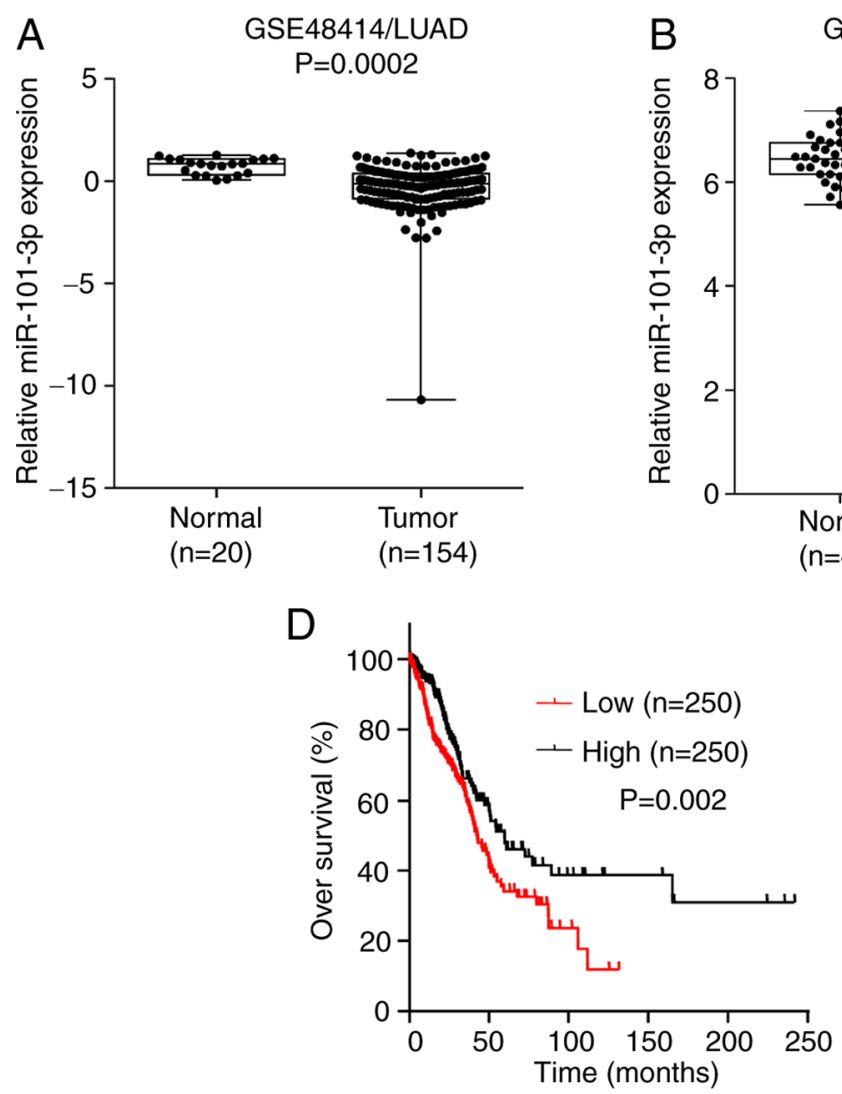
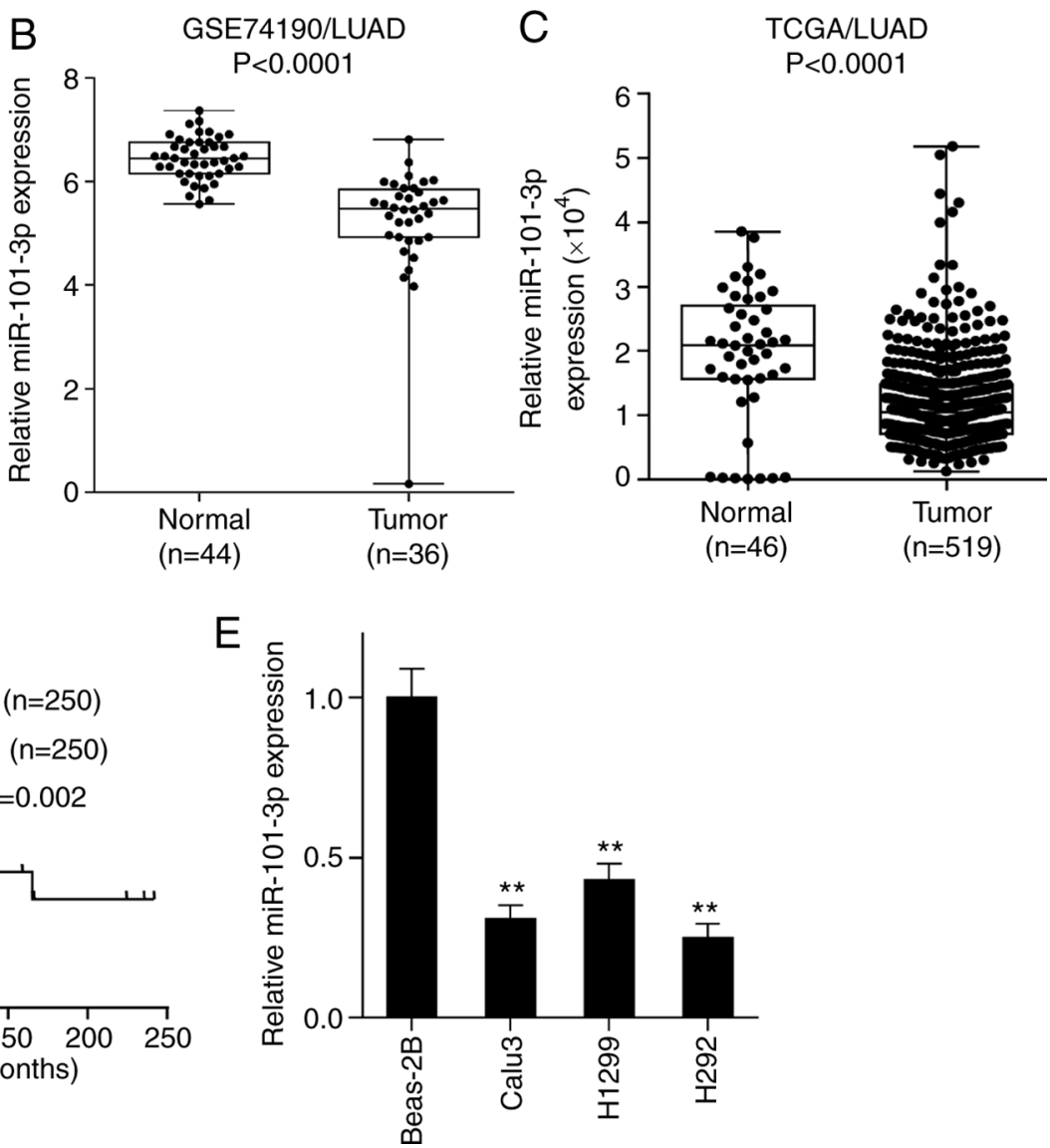

Figure 1. miR-101-3p expression is lower in patients with LUAD tissues and associated with poor prognosis of patients with LUAD. (A-C) Expression of miR-101-3p in LUAD tissues and normal lung tissues in datasets from the Gene Expression Omnibus datasets and the TCGA database. (D) Analysis of the overall survival of patients with LUAD from TCGA. (E) Expression of miR-101-3p in LUAD cell lines Calu3, H1299 and H292 and the normal lung epithelial cell line BEAS-2B was detected by reverse transcription quantitative PCR. ${ }^{* *} \mathrm{P}<0.01$ vs. BEAS-2B cell line. LUAD, lung adenocarcinoma; TCGA, The Cancer Genome Atlas; miR, microRNA.

at $48 \mathrm{~h}$ after irradiation. The expression of the pro-apoptotic protein Bax was increased and that of the anti-apoptotic Bcl-2 was decreased in cells overexpressing miR-101-3p compared with control cells (Fig. 2J). These findings indicated that miR-101-3p may promote the radiation-induced cell apoptosis by increasing the expression of Bax and decreasing the expression of Bcl-2 in LUAD cells. Taken together, these results suggested that miR-101-3p may enhance the radiosensitivity of LUAD cells by attenuating the DNA damage repair ability and regulating the expression of apoptosis-associated proteins.

BIRC5 is a direct target of miR-101-3p. To explore the regulatory mechanism of miR-101-3p on the radiosensitivity of LUAD cells, the Starbase was used to predict the possible targets of miR-101-3p. Among the predicted genes, BIRC5 was selected as a candidate target for its essential roles in regulating the radiosensitivity of cancer cells (26). Subsequently, analysis of the TCGA database demonstrated that the expression of BIRC5 in LUAD tissues was significantly higher than in normal lung tissue, and that miR-101-3p expression in LUAD tissues was negatively correlated with BIRC5 expression level (Fig. 3B and C). Furthermore, all patients with TCGA/LUAD were separated into low and high expression groups based on median value of BIRC5 expression (8.17), and it was demonstrated that in contrast to the impact of miR-101-3p on patient survival, patients with LUAD with high BIRC5 expression had a poorer prognosis compared with patients with a low BIRC5 expression (Fig. 3D). In addition, analysis of patient overall survival (Fig. 3E) by Kaplan-Meier Plotter online tool indicated that BIRC5 high expression level was significantly associated with a shorter overall survival of patients with lung cancer who received radiotherapy.

To validate the prediction that miR-101-3p could bind to the 3'-UTR of BIRC5 (Fig. 3A), a luciferase reporter assay was performed in LUAD cells. The results demonstrated that the luciferase activity in LUAD cells co-transfected with miR-101-3p and WT-BIRC5 was significantly decreased compared with control group, whereas no change was observed in LUAD cells co-transfected with miR-101-3p and MUT-BIRC5 (Fig. 3F and G). In addition, the mRNA and protein expression of BIRC5 in LUAD cells following transfection with miR-101-3p or miR-NC was detected, and the results demonstrated that miR-101-3p overexpression significantly decreased the expression of BIRC5 at mRNA and protein levels in LUAD cells (Fig. 3H and I). Taken together, these findings suggested that BIRC5 may be a direct target of miR-101-3p.

BIRC5 has a role in the miR-101-3p-mediated radiosensitivity of LUAD cells. As BIRC5 was demonstrated as a downstream target of miR-101-3p, we hypothesized that BIRC5 might 
A

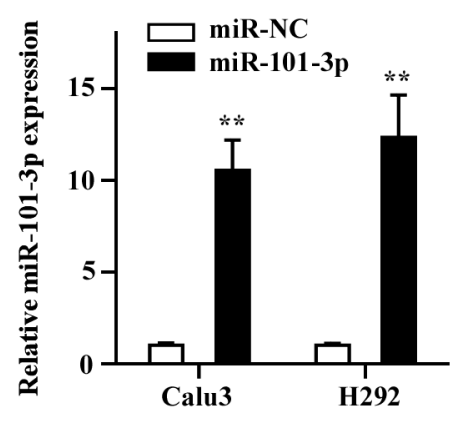

B

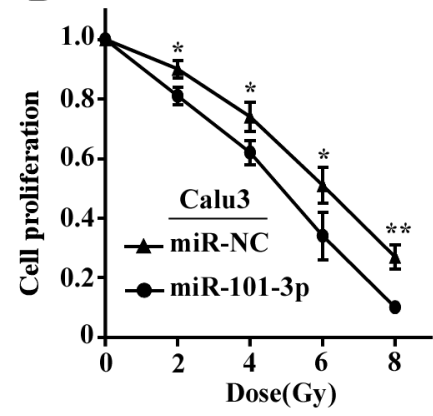

C

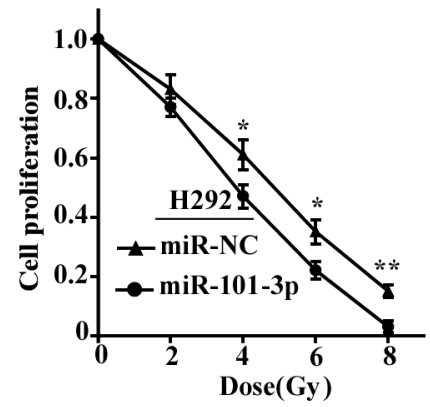

F

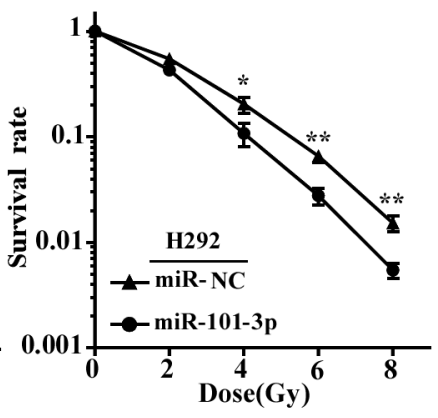

G

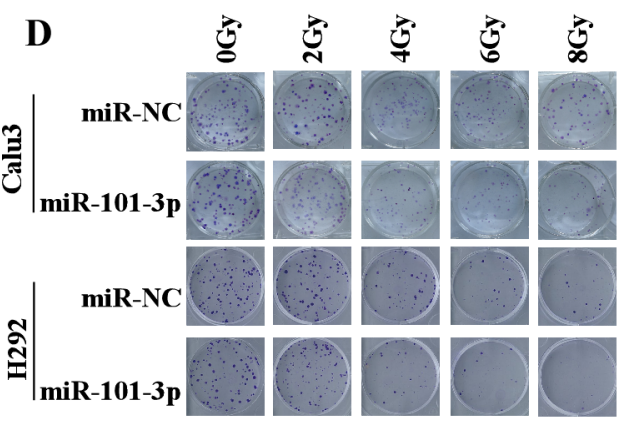

H
E

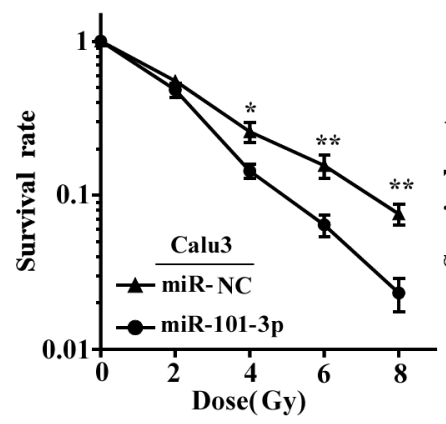

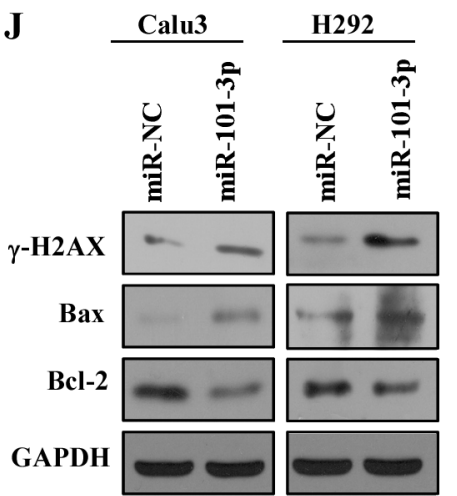
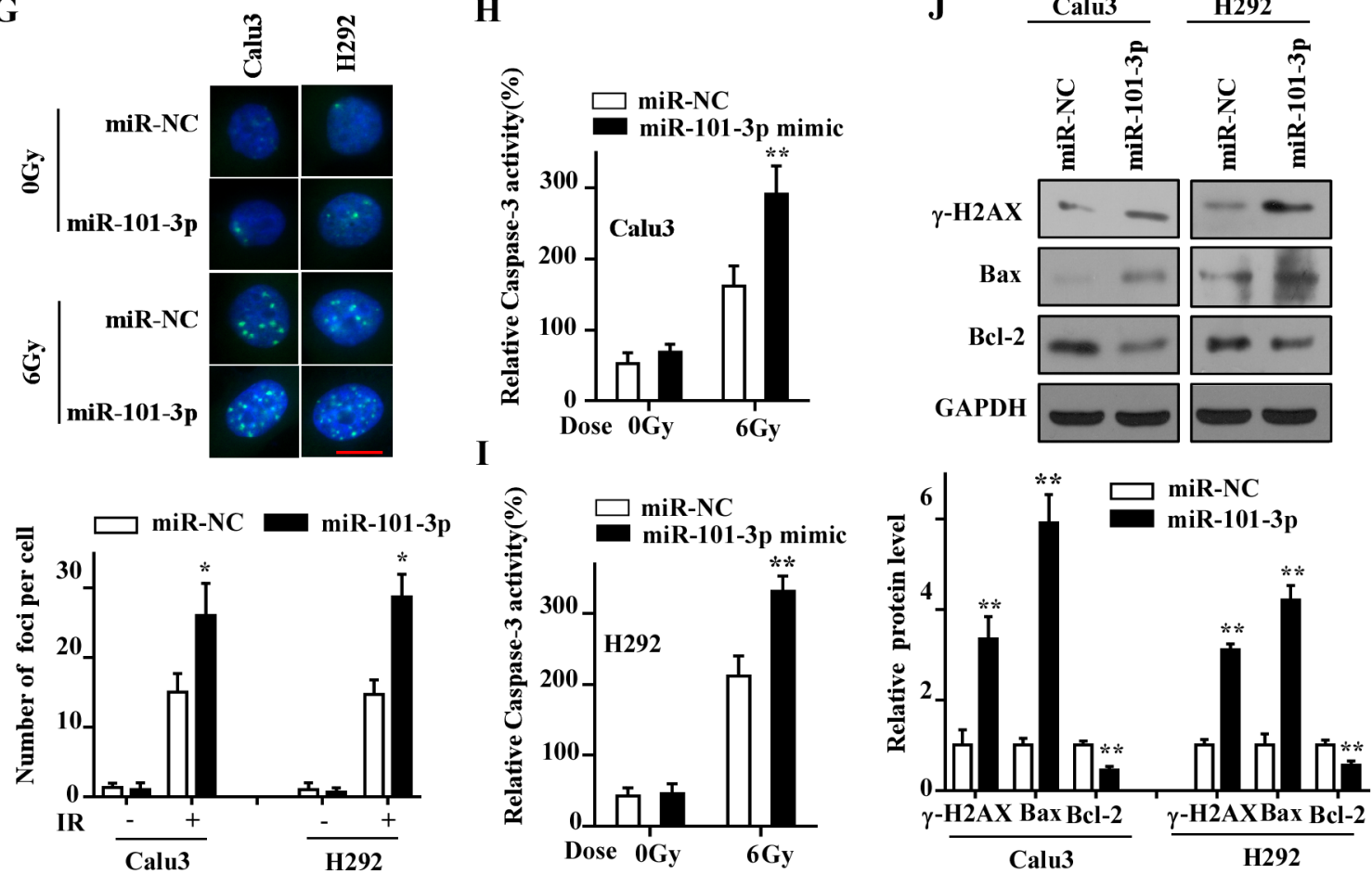

Figure 2. Overexpression of miR-101-3p sensitizes LUAD cells to IR. (A) Expression of miR-101-3p in cells transfected with miR-101-3p mimics assessed by reverse transcription quantitative PCR. (B and C) Proliferation of LUAD cells treated with 0, 2, 4, 6 and 8 Gy of IR was determined by Cell Counting Kit-8 assay. (D-F) Colony forming assay indicated that transfection of miR-101-3p mimics enhanced radiosensitivity of LUAD cells compared with control group cells after treatment with $0,2,4,6$ and 8 Gy of IR. (G) Cells were treated with 6 Gy IR and stained with antibody against $\gamma$-H2AX (scale bar $=10 \mu$ m). (H and I) Caspase-3 activity was detected in cells exposed to IR. (J) Protein expression of $\gamma$-H2AX, Bax, Bcl-2 and GAPDH in LUAD cells determined by western blotting. ${ }^{*} \mathrm{P}<0.05$ and ${ }^{* *} \mathrm{P}<0.01$ vs. miR-NC. $\gamma$-H2AX, $\gamma$-H2A histone family member X; LUAD, lung adenocarcinoma; miR, microRNA; IR, ionizing radiation; $\mathrm{NC}$, negative control.

be responsible for miR-101-3p-mediated radiosensitivity of LUAD cells. Rescue assays were therefore conducted to verify this hypothesis. First, the expression efficiency of BIRC5 plasmid in LUAD cells was verified by RT-qPCR (Fig. S1). Then, the results from RT-qPCR and western blotting indicated that BIRC5 was significantly upregulated at both mRNA and protein levels in LUAD cells co-transfected with miR-101-3p and BIRC5 compared with miR-101-3p and vector co-transfection (Fig. 4A and B). Furthermore, CCK- 8 and colony formation assays demonstrated that restoration of BIRC5 markedly alleviated miR-101-3p-mediated radiosensitivity of LUAD cells 
A WT BIRC5 3'UTR: 5'...GUCCCUGGCUCCUCUACUGUU...3'<smiles></smiles>

has-miR-101-3p : 3'...AAGUCAAUAGUGUCAUGACAU...5'

MUT BIRC5 3'UTR : 5'...GUCCCUGGCUCCUCAUGACAU....3'

B

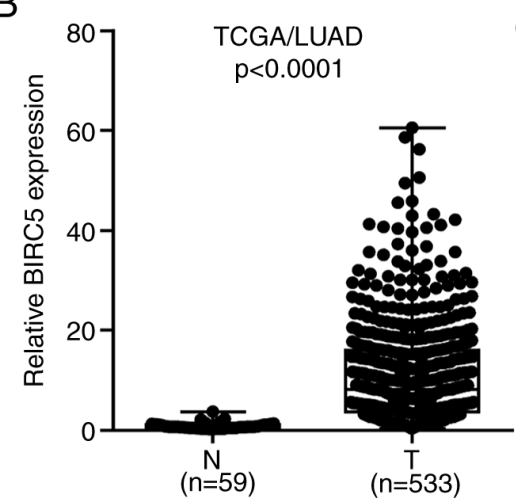

E

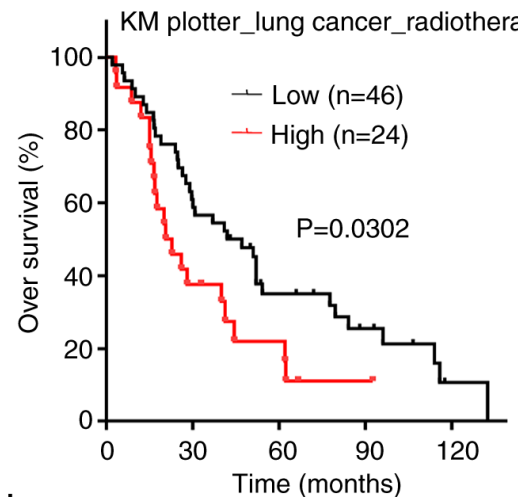

$\mathrm{H}$

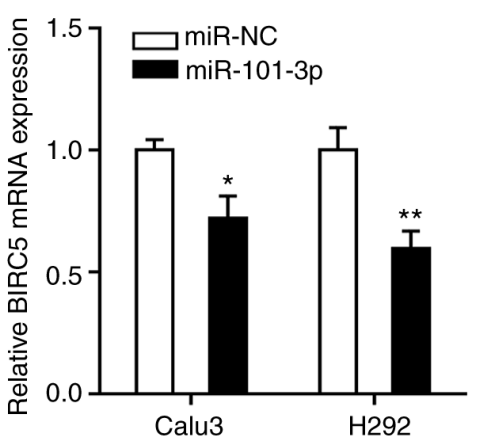

C

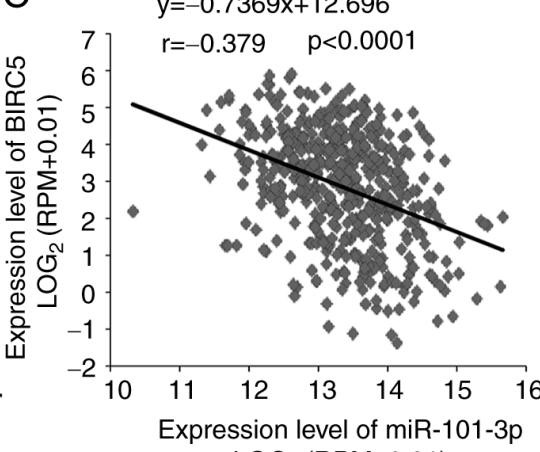

$\mathrm{LOG}_{2}(\mathrm{RPM}+0.01)$

$\mathrm{F}$

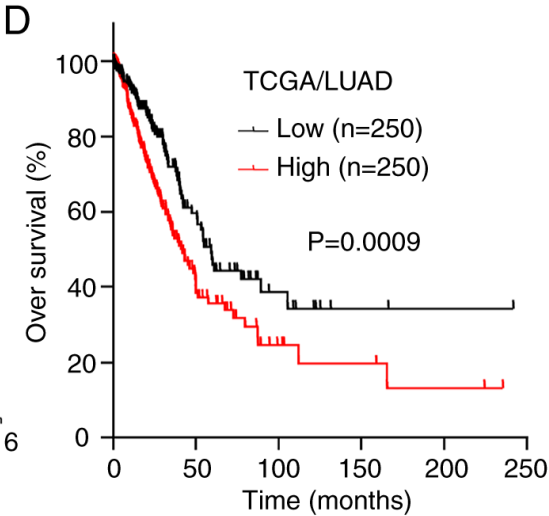

G

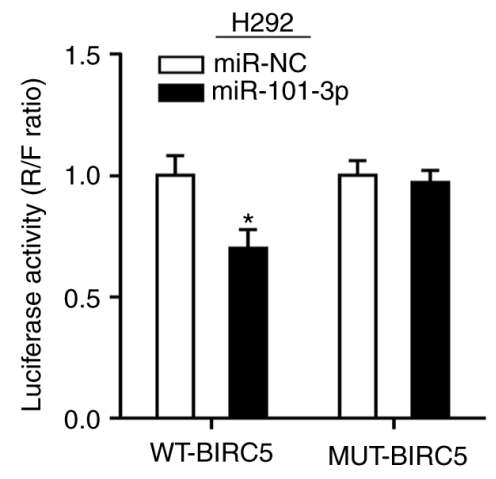

I

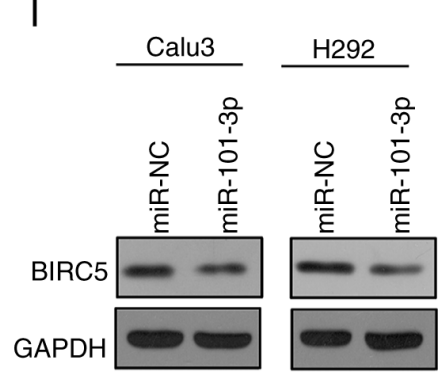

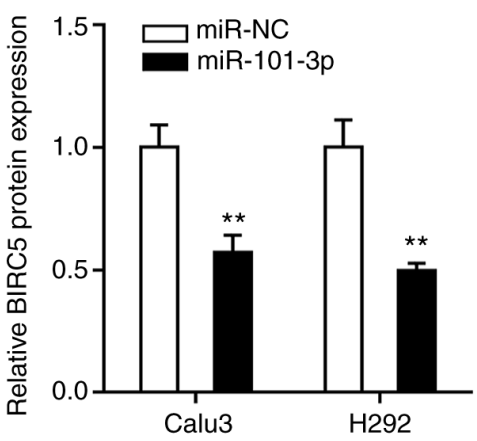

Figure 3. BIRC5 is a direct target of miR-101-3p. (A) WT and MUT binding sites for miR-101-3p in the 3'-UTR of BIRC5. (B) Expression level of BIRC5 in LUAD tissues and normal lung tissues in datasets from TCGA database. (C) Correlation analysis between BIRC5 and miR-101-3p expression levels in the tissues of patients with LUAD from TCGA. (D) Analysis of overall survival of patients with LUAD from TCGA. (E) Analysis of the overall survival of patients with LUAD who received radiotherapy using the Kaplan-Meier Plotter online tool. ( $F$ and G) LUAD cells were co-transfected with WT-BIRC5 or MUT-BIRC5 and miR-101-3p mimic or miR-NC. Luciferase activity was assessed after $48 \mathrm{~h}$. (H and I) BIRC5 mRNA and protein expression in LUAD cells. "P<0.05 and ${ }^{* *} \mathrm{P}<0.01$ vs. miR-NC. BIRC5, baculoviral IAP repeat containing 5; miR, microRNA; NC, negative control; LUAD, lung adenocarcinoma; WT, wild-type; MUT, mutant; TCGA, The Cancer Genome Atlas.

(Fig. 4C-G). BIRC5 overexpression also significantly attenuated the effects on caspase-3 activity following miR-101-3p overexpression in LUAD cells after irradiation (Fig. 4H and I). In addition, the DNA repair ability of LUAD cells was also restored after BIRC5 overexpression (Fig. 4J). Taken together, these findings supported the hypothesis that BIRC5 may serve a crucial role in the miR-101-3p-mediated radiosensitivity of LUAD cells.

\section{Discussion}

Radiotherapy is an important treatment for LUAD; however, radioresistance has impacted the survival of patients (27). The abnormal proliferation, anti-apoptotic process and DNA damage repair in cancer cells are considered to be the main mechanisms of radioresistance (28). Previous studies have indicated that miRNAs can serve as important regulators in 
A

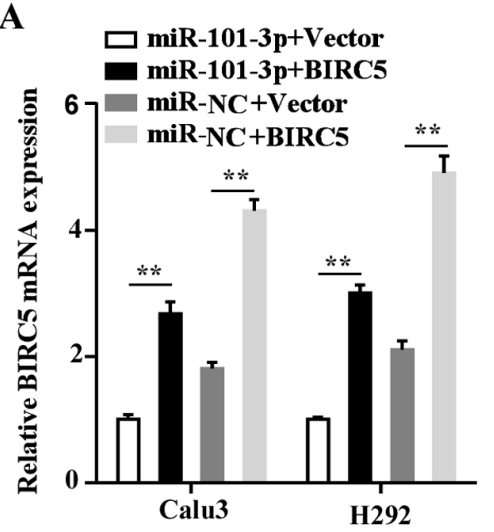

C

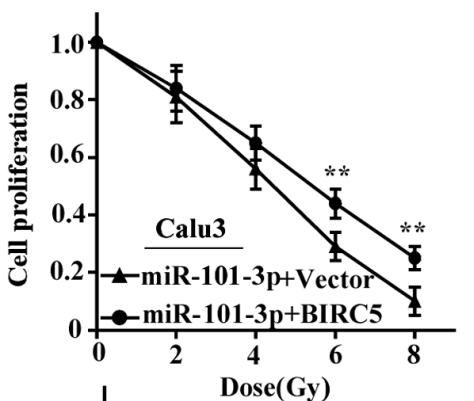

F

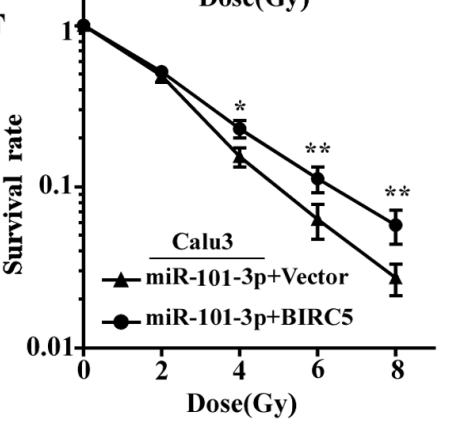

I

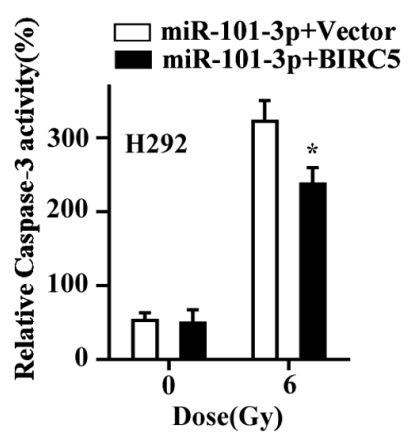

B

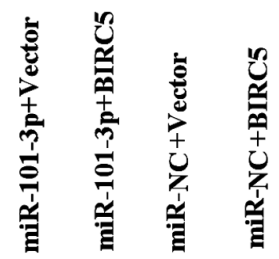

苞| $\begin{gathered}\text { BIRC5 } \\ \text { GAPDH }\end{gathered}$

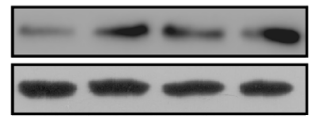

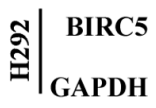

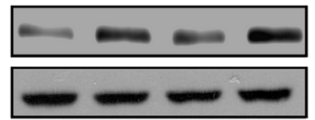

D
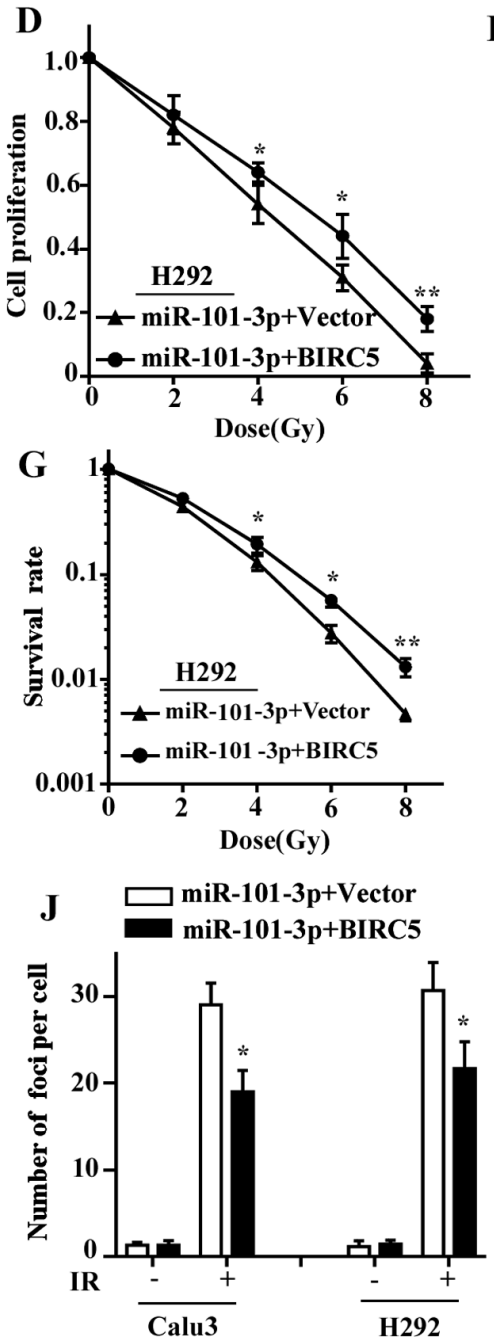

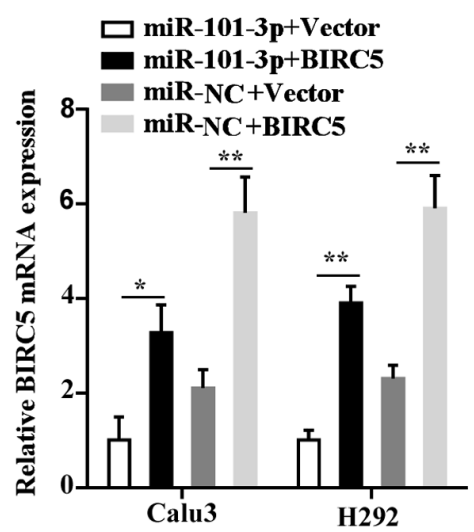

E

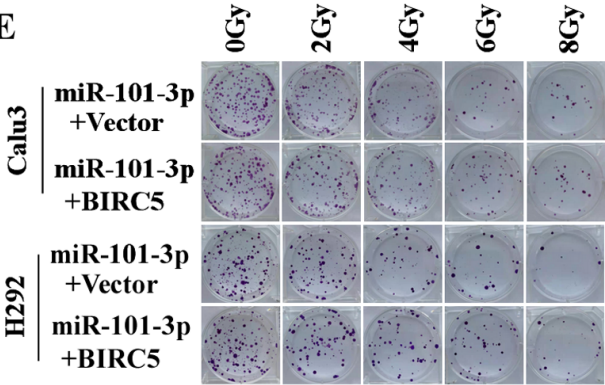

H
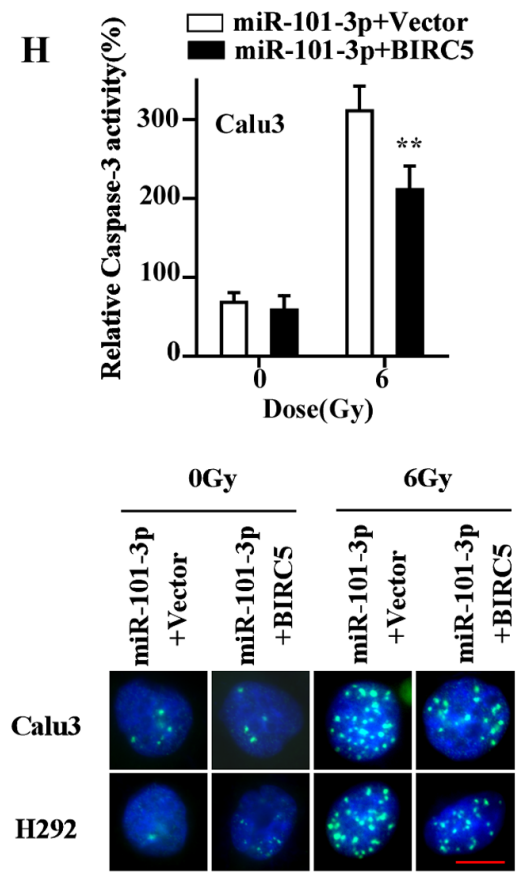

Figure 4. BIRC5 has a crucial role in miR-101-3p-mediated radiosensitivity of LUAD cells. (A and B) BIRC5 mRNA and protein expression in LUAD cells co-transfected with BIRC5 or vector plasmids and miR-101-3p mimic or miR-NC. (C and D) Proliferation of LUAD cells treated with 0, 2, 4, 6 and 8 Gy of IR was determined by Cell Counting Kit-8 assay. (E-G) Colony formation assay showed that restoration of BIRC5 markedly alleviated miR-101-3p-mediated radiosensitivity of LUAD cells. (H and I) Restoration of BIRC5 significantly attenuated the enhanced effects on caspase-3 activity following miR-101-3p overexpression in LUAD cells in response to irradiation. ( $\mathrm{J})$ DNA repair ability of LUAD cells was restored by BIRC5 overexpression (scale bar $=10 \mu$ m). ${ }^{*} \mathrm{P}<0.05$ and ${ }^{* *} \mathrm{P}<0.01$ vs. miR-101-3p + vector group or miR-NC +vector group. BIRC5, baculoviral IAP repeat containing 5; LUAD, lung adenocarcinoma; NC, negative control; miR, microRNA.

radiosensitivity by interacting with cancer-related genes $(29,30)$. Therefore, the identification of miRNAs and their target genes associated with radioresistance is crucial in cancer treatment. The results from the present study demonstrated that miR-101-3p may serve an important role in regulating LUAD cell radiosensitivity. In particular, BIRC5 was predicted and confirmed to be a direct target gene of miR-101-3p, which is involved in the radiosensitivity of LUAD cells.

miR-101-3p has been reported to be frequently downregulated and to exhibit antitumorigenic properties in various types of cancer $(27,31,32)$. The results from the present study demonstrated that miR-101-3p expression was lower 
in LUAD tissues compared with normal lung tissues and considered as a poor prognostic factor for patients, according to analysis of GEO and TCGA data. These findings suggested that miR-101-3p may function as a tumor suppressor in LUAD. Subsequently, we hypothesized that miR-101-3p might participate in the regulation of LUAD treatment sensitivity. miR-101-3p expression has been reported to be negatively correlated with chemoresistance in several types of malignancy, such as gastric cancer and colon cancer (33-35). However, the role of miR-101-3p in LUAD radiosensitivity remains unknown. The present study demonstrated that miR-101-3p could sensitize LUAD cells to irradiation via targeting BIRC5, suggesting that the regulation of miR-101-3p expression and its target BIRC5 may help the development novel radio-sensitizers.

BIRC5 is the smallest member of the IAP family and has attracted attention due to its abnormally increased expression in a variety of human cancers, its prognostic relevance and its prominent role in the regulation of cancer cell apoptosis and proliferation $(36,37)$. BIRC5 has also been reported to participate in the repair of radiation-induced DNA damage, thereby affecting cancer cell sensitivity to radiotherapy and chemotherapy. For example, overexpression of nuclear BIRC5 can enhance DNA repair in glioblastoma cells, leading to radioresistance in glioblastoma (15). Furthermore, BIRC5 depletion decreases the expression of DNA damage repair-related genes, sensitizing therefore cancer cells to PARP inhibition (26). In NSCLC, BIRC5 has also been demonstrated to promote DNA damage response and radiotherapy resistance (38).

In the present study, BIRC5 was predicted to be a target of miR-101-3p. Subsequently, the results demonstrated that BIRC5 was highly expressed in LUAD tissues compared with normal lung tissues and was considered as a poor prognostic factor in patients, according to TCGA data analysis. Furthermore, BIRC5 and miR-101-3p expression levels were negatively correlated, and their expression had opposite effects on patient survival. In addition, analysis of the overall survival of patients with LUAD using Kaplan-Meier Plotter online database indicated that BIRC5 expression level was associated with the overall survival of patients who received radiotherapy. Taken together, these findings suggested that miR-101-3p may likely affect patients survival prognosis and radiotherapy efficacy by targeting BIRC5. Indeed, this study demonstrated that BIRC5 was a direct target site for miR-101-3p, and that restoring BIRC5 significantly inhibited miR-101-3p-mediated decline in proliferation, increased apoptosis and impaired DNA repair capacity in LUAD cells following radiation exposure. BIRC5 may therefore serve a crucial role in miR-101-3p-mediated radiosensitivity of LUAD cells.

In summary, the present study demonstrated that miR-101-3p could sensitize LUAD cells to irradiation via targeting BIRC5. These findings may provide a new mechanism for radioresistance and help the development of novel strategies to treat patients with LUAD.

\section{Acknowledgements}

Not applicable.

\section{Funding}

No funding was received.

\section{Availability of data and materials}

The datasets used and/or analyzed during the current study are available from the corresponding author on reasonable request.

\section{Authors' contributions}

$\mathrm{XM}$ and YS conducted the cell culture, cell transfection, CCK-8 assay, colony formation assay, caspase-3 activity assay, and were major contributors in writing the manuscript. XM and YM conducted the RT-qPCR, western blot, immunofluorescence staining, luciferase reporter assay and performed the statistical analyses. SL performed public platform database analysis and revised the manuscript. YS contributed to the study design. XM and YM confirm the authenticity of all the raw data. All authors read and approved the final version of the manuscript.

\section{Ethics approval and consent to participate}

Not applicable.

\section{Patient consent for publication}

Not applicable.

\section{Competing interests}

The authors declare that they have no competing interests.

\section{References}

1. Siegel RL, Miller KD and Jemal A: Cancer statistics, 2016. CA Cancer J Clin 66: 7-30, 2016.

2. Chen J, Xu Y, Tao L, Pan Y, Zhang K, Wang R, Chen LB and Chu X: miRNA-26a contributes to the acquisition of malignant behaviors of doctaxel-resistant lung adenocarcinoma cells through targeting EZH2. Cell Physiol Biochem 41: 583-597, 2017.

3. Siegel R, Naishadham D and Jemal A: Cancer statistics, 2012. CA Cancer J Clin 62: 10-29, 2012.

4. Jonas S and Izaurralde E: Towards a molecular understanding of microRNA-mediated gene silencing. Nat Rev Genet 16: 421-433, 2015.

5. Rupaimoole R and Slack FJ: MicroRNA therapeutics: Towards a new era for the management of cancer and other diseases. Nat Rev Drug Discov 16: 203-222, 2017.

6. Di Leva G, Garofalo M and Croce CM: MicroRNAs in cancer. Annu Rev Pathol 9: 287-314, 2014.

7. Huang T, Yin L, Wu J, Gu JJ, Wu JZ, Chen D, Yu HL, Ding K, Zhang N, Du MY, et al: MicroRNA-19b-3p regulates nasopharyngeal carcinoma radiosensitivity by targeting TNFAIP3/NF- $\kappa \mathrm{B}$ axis. J Exp Clin Cancer Res 35: 188, 2016.

8. Chen W, Song J, Bian H, Yang X, Xie X, Zhu Q, Qin C and Qi J: The functions and targets of miR-212 as a potential biomarker of cancer diagnosis and therapy. J Cell Mol Med 24: 2392-2401, 2020.

9. Wu X, Zhou J, Wu Z, Chen C, Liu J, Wu G, Zhai J, Liu F and Li G: miR-101-3p suppresses HOX transcript antisense RNA (HOTAIR)-induced proliferation and invasion through directly targeting SRF in gastric carcinoma cells. Oncol Res 25: 1383-1390, 2017.

10. Hou Y, Li L, Ju Y, Lu Y, Chang L and Xiang X: miR-101-3p regulates the viability of lung squamous carcinoma cells via targeting EZH2. J Cell Biochem 118: 3142-3149, 2017.

11. Li B, Xie D and Zhang H: MicroRNA-101-3p advances cisplatin sensitivity in bladder urothelial carcinoma through targeted silencing EZH2. J Cancer 10: 2628-2634, 2019.

12. Yamamoto H, Ngan CY and Monden M: Cancer cells survive with survivin. Cancer Sci 99: 1709-1714, 2008. 
13. Pennati $\mathrm{M}$, Folini $\mathrm{M}$ and Zaffaroni $\mathrm{N}$ : Targeting survivin in cancer therapy. Expert Opin Ther Targets 12: 463-476, 2008.

14. Greve B, Sheikh-Mounessi F, Kemper B, Ernst I, Götte M and Eich HT: Survivin, a target to modulate the radiosensitivity of Ewing's sarcoma. Strahlenther Onkol 188: 1038-1047, 2012.

15. Reichert S, Rödel C, Mirsch J, Harter PN, Tomicic MT, Mittelbronn M, Kaina B and Rödel F: Survivin inhibition and DNA double-strand break repair: A molecular mechanism to overcome radioresistance in glioblastoma. Radiother Oncol 101: $51-58,2011$.

16. Erpolat OP, Gocun PU, Akmansu M, Karakus E and Akyol G: High expression of nuclear survivin and Aurora $B$ predicts poor overall survival in patients with head and neck squamous cell cancer. Strahlenther Onkol 188: 248-254, 2012.

17. Romagnoli M, Séveno C, Bataille R and Barillé-Nion S: Survivin in cancerology: Molecular aspects and therapeutic applications. Med Sci (Paris) 24: 821-827, 2008 (In French).

18. Sprenger T, Rödel F, Beissbarth T, Conradi LC, Rothe H, Homayounfar K, Wolff HA, Ghadimi BM, Yildirim M, Becker $\mathrm{H}$, et al: Failure of downregulation of survivin following neoadjuvant radiochemotherapy in rectal cancer is associated with distant metastases and shortened survival. Clin Cancer Res 17: 1623-1631, 2011.

19. Bjaanaes MM, Halvorsen AR, Solberg S, Jørgensen L, Dragani TA, Galvan A, Colombo F, Anderlini M, Pastorino U, Kure E, et al: Unique microRNA-profiles in EGFR-mutated lung adenocarcinomas. Int J Cancer 135: 1812-1821, 2014.

20. Wu KH, Zhou B, Lu SH, Feng B, Yang SG, Du WT, Gu DS, Han ZC and Liu YL: In vitro and in vivo differentiation of human umbilical cord derived stem cells into endothelial cells. J Cell Biochem 100: 608-616, 2007.

21. Yang JH, Li JH, Shao P, Zhou H, Chen YQ and Qu LH: StarBase: A database for exploring microRNA-mRNA interaction maps from Argonaute CLIP-Seq and Degradome-Seq data. Nucleic Acids Res 39 (Database Issue): D202-D209, 2011.

22. Menyhárt O, Nagy Á and Győrffy B: Determining consistent prognostic biomarkers of overall survival and vascular invasion in hepatocellular carcinoma. R Soc Open Sci 5: 181006, 2018.

23. Livak KJ and Schmittgen TD: Analysis of relative gene expression data using real-time quantitative PCR and the 2(-Delta Delta C(T)) method. Methods 25: 402-408, 2001.

24. Mariotti LG, Pirovano G, Savage KI, Ghita M, Ottolenghi A, Prise KM and Schettino G: Use of the $\gamma-\mathrm{H} 2 \mathrm{AX}$ assay to investigate DNA repair dynamics following multiple radiation exposures. PLoS One 8: e79541, 2013.

25. Zhang J, Shen L and Sun LQ: The regulation of radiosensitivity by 553 and its acetylation. Cancer Lett 363: 108-118, 2015.

26. Véquaud E, Desplanques G, Jézéquel $P$, Juin $P$ and Barillé-Nion S: Survivin contributes to DNA repair by homologous recombination in breast cancer cells. Breast Cancer Res. Treat 155: 53-63, 2016.
27. Dong Y, Zhang D, Cai M, Luo Z, Zhu Y, Gong L, Lei Y, Tan X, Zhu Q and Han S: SPOP regulates the DNA damage response and lung adenocarcinoma cell response to radiation. Am J Cancer Res 9: 1469-1483, 2019.

28. Yang S, Chen J, Guo Y, Lin H, Zhang Z, Feng G, Hao Y, Cheng J, Liang P, Chen K, et al: Identification of prognostic biomarkers for response to radiotherapy by DNA microarray in nasopharyngeal carcinoma patients. Int J Oncol 40: 1590-1600, 2012.

29. Salim H, Akbar NS, Zong D, Vaculova AH, Lewensohn R, Moshfegh A, Viktorsson K and Zhivotovsky B: miRNA-214 modulates radiotherapy response of non-small cell lung cancer cells through regulation of p38MAPK, apoptosis and senescence. Br J Cancer 107: 1361-1373, 2012.

30. Fang H, Xie J, Zhang M, Zhao Z, Wan Y and Yao Y: miRNA-21 promotes proliferation and invasion of triple-negative breast cancer cells through targeting PTEN. Am J Transl Res 9: 953-961, 2017.

31. Thu KL, Chari R, Lockwood WW, Lam S and Lam WL: miR-101 DNA copy loss is a prominent subtype specific event in lung cancer. J Thorac Oncol 6: 1594-1598, 2011.

32. Li L, Shao MY, Zou SC, Xiao ZF and Chen ZC: miR-101-3p inhibits EMT to attenuate proliferation and metastasis in glioblastoma by targeting TRIM44. J Neurooncol 141: 19-30, 2019.

33. Bao J, Xu Y, Wang Q, Zhang J, Li Z, Li D and Li J: miR-101 alleviates chemoresistance of gastric cancer cells by targeting ANXA2. Biomed Pharmacother 92: 1030-1037, 2017.

34. Chen LG, Xia YJ and Cui Y: Upregulation of miR-101 enhances the cytotoxic effect of anticancer drugs through inhibition of colon cancer cell proliferation. Oncol Rep 38: 100-108, 2017.

35. Xu F, Liao JZ, Xiang GY, Zhao PX, Ye F, Zhao Q and He XX: miR-101 and doxorubicin codelivered by liposomes suppressing malignant properties of hepatocellular carcinoma. Cancer Med 6: 651-661, 2017.

36. Altieri DC: Molecular circuits of apoptosis regulation and cell division control: The survivin paradigm. J Cell Biochem 92: 656-663, 2004

37. Altieri DC: Survivin, cancer networks and pathway-directed drug discovery. Nat Rev Cancer 8: 61-70, 2008.

38. Hu S, Qu Y, Xu X, Xu Q, Geng J and Xu J: Nuclear survivin and its relationship to DNA damage repair genes in non-small cell lung cancer investigated using tissue array. PLoS One 8: e74161, 2013.

This work is licensed under a Creative Commons Attribution-NonCommercial-NoDerivatives 4.0 International (CC BY-NC-ND 4.0) License. 